\title{
M-polynomials and degree-based topological indices of Bismuth Tri-Iodide
}

\author{
Young Chel Kwun' ${ }^{1}$, Abaid ur Rehman Virk ${ }^{2}$, Waqas Nazeer ${ }^{3, *}$ and Shin Min Kang ${ }^{4}$
}

1. Department of Mathematics, Dong A University, Korea; yckwun@dau.ac.kr

2. Department of Mathematics, University of Management and Technology, Lahore Pakistan; abaid.math@gmail.com

3. Department of Mathematics, Division of Science and Technology, University of Education Lahore Pakistan

4. Department of Mathematics and RINS, Gyeongsang National University, Jinju 52828, Korea, smkang@gnu.ac.kr

Corresponding Author: nazeer.waqas@ue.edu.pk ; +92-321-4707379

\begin{abstract}
The topological index is a numerical quantity based on the characteristics of various invariants or molecular graph. For ease of discussion, these indices are classified according to their logical derivation from topological invariants rather than their temporal development. Degree based topological indices depends upon the degree of vertices. This paper computes degree based topological indices of Bismuth Tri-Iodide chains and sheets with the help of M-polynomial.
\end{abstract}

Keywords: topological index, Bismuth Tri-Iodide, Molecular graph, Zagreb index, Randic index, Mpolynomial.

\section{Introduction}

$\mathrm{BiI}_{3}$ is an inorganic compound which is the result of the reaction of iodine and bismuth, which inspired the enthusiasm for subjective inorganic investigations [1]. $\mathrm{BiI}_{3}$ is an excellent inorganic compound and is very useful in qualitative inorganic analysis [2].

It has been proven that $B i$-doped glass optical strands are one of the most promising dynamic laser media. Different kinds of Bi-doped fiber strands have been created and have been used to construct Bi-doped fiber lasers and optical loudspeakers [3].

Layered $\mathrm{BiI}_{3}$ gemstones are considered to be a three-layered stack structure in which a plane of bismuth atoms is sandwiched between iodide particle planes to form a continuous $I-B i-I$ plane [4].

The periodic superposition of the three layers forms diamond-shaped $\mathrm{BiI}_{3}$ crystals with $R-3$ symmetry [5,6]. A progressive stack of $I-B i-I$ layers forms a hexagonal structure with symmetry [7]. A jewel of $\mathrm{BiI}_{3}$ has been integrated in [8]. 


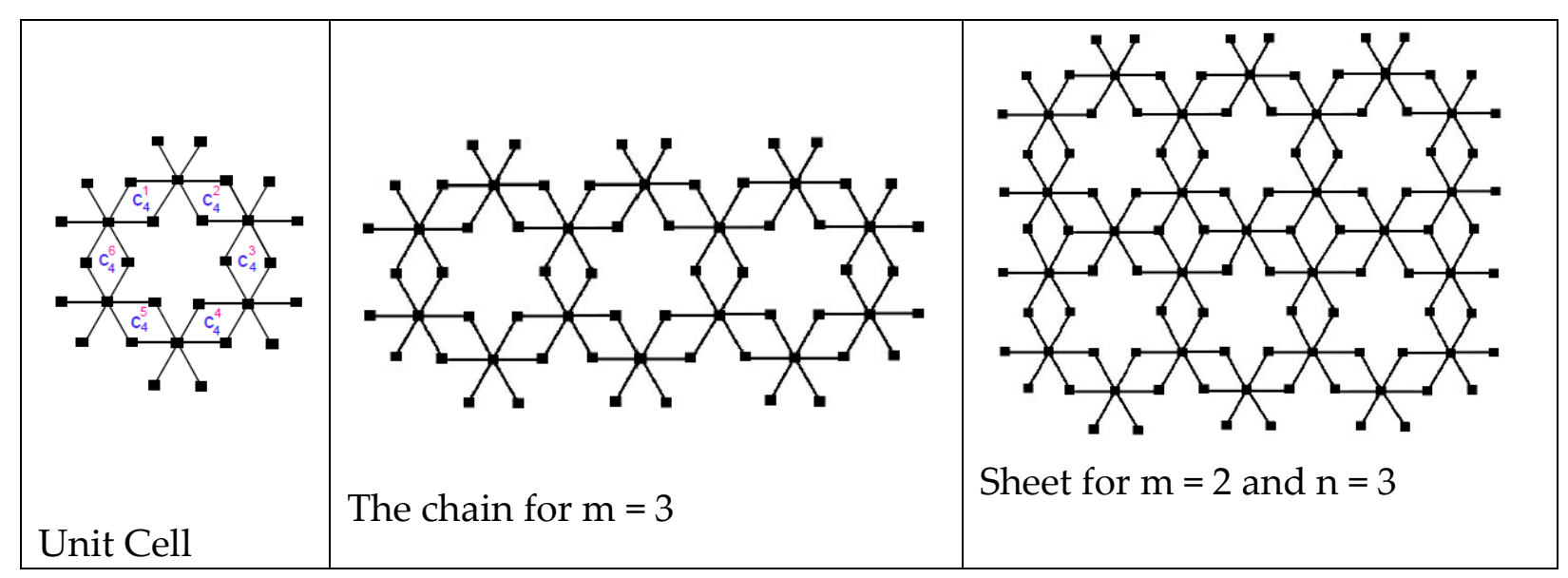

Figure 1 bismuth tri-iodide

In the unit cell (figure 1), Main cycles are $C_{4}^{1}, C_{4}^{2}$ central cycles are $C_{4}^{3}, C_{4}^{6}$ and Base cycles are $C_{4}^{4}, C_{4}^{5}$

Given its enormous application in the science of impurity-free and connection, graph theory is a multidimensional topic. It is feasible to display and plan crystal structures, complex systems, and synthesis charts. There are many compounds that are organic and inorganic compounds that can be used in commercial, industrial and laboratory environments as well as in everyday life. There is a relationship between the synthesis mixture and its atomic structure. Graph theory is an effective field of arithmetic and has a huge range of applications in many scientific fields such as chemistry, software engineering, electrical and electronics. Chemical graph theory is other branches of science in which graphs are used to show the mixture graphically using proficient instruments.

The physical structure of a strong material usually depends on the action of atoms, particles, or atoms that make up a strong bond between them. The crystal structure, also known as crystal material or crystal strength, is made of a unit cell, which is organized in $3 \mathrm{D}$ on the grid. The scheme of atomic or crystalline materials is crucial for determining the behavior and properties of materials such as metals, composites, and art materials. Cells are the smallest auxiliary units that can clarify the gem structure (unit cell). The redundancy of the cell creates the entire structure.

Mathematical chemistry provides tools such as polynomials and functions to capture information hidden in the symmetry of molecular graphs and thus predict properties of compounds without using quantum mechanics. A topological index isa numerical parameter of a graph and depicts its topology. It describes the structure of molecules numerically and are used in the development of qualitative structure activity relationships (QSARs). Most commonly known invariants of such kinds are degree- 
based topological indices. These are actually the numerical values that correlate the structure with various physical properties, chemical reactivity and biological activities [9-13]. It is an established fact that many properties such as heat of formation, boiling point, strain energy, rigidity and fracture toughness of a molecule are strongly connected to its graphical structure.

Hosoya polynomial, (Wiener polynomial), [14] plays a pivotal role in distance-based topological indices. A long list of distance-based indices can be easily evaluated from Hosoya polynomial. A similar breakthrough was obtained recently by Klavzar et. al. [15], in the context of degree-based indices. Authors in [15] introduced M-polynomial in, 2015, to play a role, parallel to Hosoya polynomial to determine closed form of many degree-based topological indices [16-20]. The real power of M-polynomial is its comprehensive nature containing healthy information about degree-based graph invariants. These invariants are calculated on the basis of symmetries present in the $2 \mathrm{~d}-$ molecular lattices and collectively determine some properties of the material under observation.

In this article, we compute general form of M-polynomial for Bismuth Tri-Iodide chains and Bismuth Tri-Iodide sheets. Then we derive closed forms of many degree-based topological indices for these Bismuth Tri-Iodide. Zagreb indices and particular cases of Randic index was computed in [1].

\section{Basic definitions and Literature Review}

Throughout this article, we assume $G$ to be a connected graph, $V(G)$ and $E(G)$ are the vertex set and the edge set respectively and $d_{v}$ denotes the degree of a vertex $v$.

Definition 1. [15] The M-polynomial of $\mathrm{G}$ is defined as: $M(G, x, y)=\sum_{\delta \leq i \leq j \leq \Delta} m_{i j}(G) x^{i} y^{j}$ where $\delta=\operatorname{Min}\left\{d_{v} \mid v \in \mathrm{V}(\mathrm{G})\right\}, \Delta=\operatorname{Max}\left\{d_{v} \mid v \in \mathrm{V}(\mathrm{G})\right\}$, and $m_{i j}(G)$ is the edge $v u \in E(G)$ such that where $\leq j$.

Wiener index and its various applications are discussed in [21-23] . Randic' index, $R_{-1 / 2}(G)$, is introduced by Milan Randic' in 1975 defined as: $R_{-1 / 2}(G)=\sum_{u v \in E(G)} \frac{1}{\sqrt{d_{u} d_{v}}}$. For general detains about $R_{-1 / 2}(G)$ and its generalized Randic' index, $R_{\alpha}(G)=\sum_{u v \in E(G)} \frac{1}{\left(d_{u} d_{v}\right)^{\alpha}}$, please see [25-29]. 
and the inverse Randic' index is defined as $R R_{\alpha}(G)=\sum_{u v \in E(G)}\left(d_{u} d_{v}\right)^{\alpha}$. Clearly $R_{-1 / 2}(G)$ is a special case of $R_{\alpha}(G)$ when $\alpha=-\frac{1}{2}$. This index has many applications in diverse areas. Many papers and books such as [30-32] are written on this topological index as well. Gutman and Trinajstic' introduced two indices defined as: $M_{1}(G)=\sum_{u v \in E(G)}\left(d_{u}+d_{v}\right)$ and $M_{2}(G)=\sum_{u v \in E(G)}\left(d_{u} \times d_{v}\right)$. Thesecond modified Zagreb index is defined as: ${ }^{m} M_{2}(G)=\sum_{u v \in E(G)} \frac{1}{d(u) d(v)}$. We refer [38-32] to the readers for comprehensive details of these indices. Other famous indices are Symmetric division index: $\operatorname{SDD}(G)=$ $\sum_{u \in E(G)}\left\{\frac{\min \left(d_{u}, d_{v}\right)}{\max \left(d_{u}, d_{v}\right)}+\frac{\max \left(d_{u}, d_{v}\right)}{\min \left(d_{u}, d_{v}\right)}\right\}$ harmonic index defined $H(G)=\sum_{v u \in E(G)} \frac{2}{d_{u}+d_{v}}$. Inverse sum index: $I(G)=\sum_{v u \in E(G)} \frac{d_{u} d_{v}}{d_{u}+d_{v}}$. and augmented Zagreb index: $A(G)=\sum_{v u \in E(G)}\left\{\frac{d_{u} d_{v}}{d_{u}+d_{v}-2}\right\}^{3}$, $[43,44]$.

Tables presented in [15-19] relates some of these well-known degree-based topological indices with M-polynomial with following reserved notations

$D_{x}=x \frac{\partial(f(x, y)}{\partial x}, \mathcal{D}_{y}=y \frac{\partial(f(x, y)}{\partial y} \mathcal{S}_{x}=\int_{0}^{x} \frac{f(t, y)}{t} d t S_{y}=\int_{0}^{y} \frac{f(x, t)}{t} d t ? \mathcal{J}(f(x y))=f(x, x)$

$Q_{\alpha}(f(x, y))=x^{\alpha} f(x, y)$.

\section{Computational Results}

In this section we give our computational results.

\section{Bismuth Tri-Iodide Chain}

Theorem 1. Let $G$ be the graph of Bismuth Tri-Iodide Chain $m-B i I_{3}$. Then $\mathrm{M}-$ Polynomial of $m-\mathrm{BiI}_{3}$ is $M\left(m-B i I_{3}, x y\right)=(4 m+8) x y^{6}+(20 m+4) x^{2} y^{6}$.

Proof: Let $G$ be the graph of $m-\mathrm{BiI}_{3}$ bismuth tri-iodide chain.

The edge set of $m-\mathrm{BiI}_{3}$ has following two partitions [1], 


$$
\begin{gathered}
E_{1}=E_{\{1,6\}}=\left\{e=u v \in E(G) \mid d_{u}=1, d_{v}=\right\}, \\
E_{\{2,6\}}=\left\{e=u v \in E(G) \mid d_{u}=2, d_{v}=\right\},
\end{gathered}
$$

Now

$\left|E_{1}(G)\right|=4 m+8$

$\left|E_{2}(G)\right|=20 m+4$.

Thus the M-polynomial of $m-\mathrm{BiI}_{3}$ is:

$$
\begin{aligned}
M(G ; x, y) & =\sum_{i \leq j} m_{i j}(G) x^{i} y^{j} \\
& =\sum_{1 \leq 6} m_{16}(G) y^{6}+\sum_{2 \leq 6} m_{26}(G) x^{2} y^{6} \\
& =\sum_{u v \in E_{1}} m_{16}(G) x y^{6}+\sum_{u v \in E_{2}} m_{26}(G) x^{2} y^{6} \\
& =\left|E_{1}(G)\right| x y^{6}+\left|E_{2}(G)\right| x^{2} y^{6} \\
& =(4 m+8) x y^{6}+(20 m+4) x^{2} y^{6}
\end{aligned}
$$

Proposition 2. Let $\mathrm{G}$ be the graph of $m-B i I_{3}$. Then

1. $M_{1}(G)=4(47 m+22)$.

2. $M_{2}(G)=8(33 m+12)$.

3. ${ }^{m} M_{2}(G)=\frac{7}{3} m+\frac{5}{3}$.

4. $R_{\alpha}(G)=4 \cdot 6^{\alpha}\left(1+2^{\alpha} \cdot 5\right) m+4 \cdot 6^{\alpha}\left(2+2^{\alpha}\right)$.

5. $R R_{\alpha}(G)=\frac{4}{6^{\alpha}}\left(1+\frac{5}{2^{\alpha}}\right) m+\frac{4}{6^{\alpha}}\left(2+\frac{1}{2^{\alpha}}\right)$.

6. $\operatorname{SSD}(G)=\frac{274}{3} m+\frac{188}{3}$.

7. $H(G)=\frac{43}{7} m+\frac{23}{7}$.

8. $I(G)=\frac{234}{7} m+\frac{90}{7}$.

9. $A(G)=\frac{20864}{125} m+\frac{5728}{125}$.

Proof: Let

$M(G, x y)=f(x, y)=(4 m+8) x y^{6}+(20 m+4) x^{2} y^{6}$.

Then 


$$
\begin{aligned}
& D_{x} f(x, y)=(4 m+8) x y^{6}+2(20 m+4) x^{2} y^{6}, \\
& D_{y} f(x, y)=6(4 m+8) x y^{6}+6(20 m+4) x^{2} y^{6}, \\
& D_{y} D_{x} f(x, y)=6(4 m+8) x y^{6}+12(20 m+4) x^{2} y 6 \\
& S_{x} S_{y}(f(x, y))=\frac{1}{6}(4 m+8) x y^{6}+\frac{1}{12}(20 m+4) x^{2} y^{6}, \\
& D_{x}^{\alpha} D_{y}^{\alpha}(f(x, y))=6^{\alpha}(4 m+8) x y^{6}+2^{\alpha} \cdot 6^{\alpha}(20 m+8) x^{2} y^{6}, \\
& S_{x}^{\alpha} S_{y}^{\alpha}(f(x, y))=\frac{1}{6^{\alpha}}(4 m+8) x y^{6}+\frac{1}{2^{\alpha} \cdot 6^{\alpha}}(20 m+4) x^{2} y^{6}, \\
& S_{y} D_{x}(f(x, y))=\frac{1}{6}(4 m+8) x y^{6}+\frac{1}{3}(20 m+4) x^{2} y^{6}, \\
& S_{x} D_{y}(f(x, y))=6(4 m+8) x y^{6}+3(20 m+4) x^{2} y^{6}, \\
& S_{x} J f(x, y)=\frac{1}{7}(4 m+8) x^{7}+\frac{1}{8}(20 m+4) x^{8}, \\
& S_{x} J D_{x} D_{y} f(x, y)=\frac{6}{7}(4 m+8) x^{7}+\frac{3}{2}(20 m+4) x^{8}, \\
& S_{x}^{3} Q_{-2} J D_{x}^{3} D_{y}^{3} f(x, y)=\frac{216}{125}(4 m+8) x^{5}+8(20 m+4) x^{6} .
\end{aligned}
$$

1. First Zagreb Index

$M_{1}(G)=\left.\left(D_{x}+D_{y}\right) f(x, y)\right|_{x=y=1}=4(47 m+22)$.

2. Second Zagreb Index $M_{2}(G)=\left.D_{y} D_{x}(f(x, y))\right|_{x=y=1}=8(33 m+12)$.

\section{Modified Second Zagreb Index}

${ }^{m} M_{2}(G)=\left.S_{x} S_{y}(f(x, y))\right|_{x=y=1}=\frac{7}{3} m+\frac{5}{3}$.

\section{Generalized Randic' Index}

$R_{\alpha}(G)=\left.D_{x}^{\alpha} D_{y}^{\alpha}(f(x, y))\right|_{x=y=1}=4 \cdot 6^{\alpha}\left(1+2^{\alpha} \cdot 5\right) m+4 \cdot 6^{\alpha}\left(2+2^{\alpha}\right)$.

\section{Inverse Randic' Index}

$$
R R_{\alpha}(G)=\left.S_{x}^{\alpha} S_{y}^{\alpha}(f(x, y))\right|_{x=y=1}=\frac{4}{6^{\alpha}}\left(1+\frac{5}{2^{\alpha}}\right) m+\frac{4}{6^{\alpha}}\left(2+\frac{1}{2^{\alpha}}\right) .
$$

\section{Symmetric Division Index}

$$
\operatorname{SSD}(G)=\left.\left(S_{y} D_{x}+S_{x} D_{y}\right)(f(x, y))\right|_{x=y=1}=\frac{274}{3} m+\frac{188}{3} \text {. }
$$

\section{Harmonic Index}

$$
H(G)=\left.2 S_{x} J(f(x, y))\right|_{x=1}=\frac{43}{7} m+\frac{23}{7} .
$$

\section{Inverse Sum Index}

$$
I(G)=S_{x} J D_{x} D_{y}(f(x, y))_{x=1}=\frac{234}{7} m+\frac{90}{7} .
$$


9. Augmented Zagreb Index $A(G)=\left.S_{x}{ }^{3} Q_{-2} J D_{x}{ }^{3} D_{y}{ }^{3}(f(x, y))\right|_{x=1}=\frac{20864}{125} m+\frac{5728}{125}$.

\section{Bismuth Tri-Iodide sheet}

Theorem 3. Let $G$ be the graph of Bismuth Tri-Iodide sheet $B_{i} I_{3}(m \times n)$. Then MPolynomial of $\mathrm{BiI}_{3}(m \times n)$ is $M(G, x y)=(4 m+4 n+4) x y^{6}+(12 m n+8 m+8 n-4) x^{2} y^{6}+(6 m n-6 n) x^{3} y^{6}$.

Proof: Let $G$ be the graph of $\mathrm{BiI}_{3}(m \times n)$ bismuth tri-iodide sheet.

The edge set of $\mathrm{BiI}_{3}(m \times n)$ has following three partitions [1],

$$
\begin{aligned}
& E_{1}=E_{\{1,6\}}=\left\{e=u v \in E(G) \mid d_{u}=1, u_{v}=\right\}, \\
& E_{2}=E_{\{2,6\}}=\left\{e=u v \in E(G) \mid d_{u}=2, u_{v}=\right\}, \\
& E_{3}=E_{\{3,6\}}=\left\{e=u v \in E(G) \mid d_{u}=3, u_{v}=\right\},
\end{aligned}
$$

Now

$$
\begin{aligned}
& \left|E_{1}(G)\right|=4 m+4 n+4, \\
& \left|E_{2}(G)\right|=12 m n+8 m+8 n-4, \\
& \left|E_{3}(G)\right|=6 m n-6 n .
\end{aligned}
$$

Thus the M-polynomial of $\mathrm{BiI}_{3}(m \times n)$ is:

$$
\begin{aligned}
M(G ; x, y) & =\sum_{i \leq j} m_{i j}(G) x^{i} y^{j} \\
& =\sum_{1 \leq 6} m_{16}(G) y^{6}+\sum_{2 \leq 6} m_{26}(G) x^{2} y^{6}+\sum_{3 \leq 6} m_{36}(G) x^{3} y^{6} \\
& =\sum_{u v \in E_{1}} m_{16}(G) x y^{6}+\sum_{u v \in E_{2}} m_{26}(G) x^{2} y^{6}+\sum_{u v \in E_{3}} m_{36}(G) x^{3} y^{6} \\
& =\left|E_{1}(G)\right| x y^{6}+\left|E_{2}(G)\right| x^{2} y^{6}+\left|E_{3}(G)\right| x^{3} y^{6} \\
& =(4 m+4 n+4) x y^{6}+(12 m n+8 m+8 n-4) x^{2} y^{6}+(6 m n-6 n) x^{3} y^{6}
\end{aligned}
$$

Proposition 4. Let $\mathrm{G}$ be the graph of $\mathrm{BiI}_{3}(m \times n)$. Then 
1. $M_{1}(G)=150 m n+92 m+38 n-4$.

2. $M_{2}(G)=252 m n+120 m+12 n-24$.

3. ${ }^{m} M_{2}(G)=\frac{4}{3} m n+\frac{4}{3} m+n+\frac{1}{3}$.

4. $R_{\alpha}(G)=6^{\alpha}(4 m+4 n+4)+2^{\alpha} \cdot 6^{\alpha}(12 m n+8 m+8 n-4)+3^{\alpha} \cdot 6^{\alpha}(6 m n-6 n)$.

5. $R R_{\alpha}(G)=\frac{1}{6^{\alpha}}(4 m+4 n+4)+\frac{1}{2^{\alpha} \cdot 6^{\alpha}}(12 m n+8 m+8 n-4)+\frac{1}{3^{\alpha} \cdot 6^{\alpha}}(6 m n-6 n)$.

6. $\operatorname{SSD}(G)=55 m n+\frac{154}{3} m+\frac{109}{3} n+\frac{34}{3}$.

7. $H(G)=\frac{13}{3} m n+\frac{22}{7} m+\frac{38}{21} n-\frac{1}{7}$.

8. $I(G)=30 m n+\frac{108}{7} m+\frac{24}{7} n-\frac{18}{7}$.

9. $A(G)=\frac{67920}{343} m n+\frac{8864}{125} m-\frac{1333648}{42875} n-\frac{3136}{125}$.

Proof: Let

$M(G, x y)=f(x, y)=(4 m+4 n+4) x y^{6}+(12 m n+8 m+8 n-4) x^{2} y^{6}+(6 m n-6 n) x^{3} y^{6}$.

Then

$$
\begin{aligned}
& D_{x} f(x, y)=(4 m+4 n+4) x y^{6}+2(12 m n+8 m+8 n-4) x^{2} y^{6}+3(6 m n-6 n) x^{3} y^{6}, \\
& D_{y} f(x, y)=6(4 m+4 n+4) x y^{6}+6(12 m n+8 m+8 n-4) x^{2} y^{6}+6(6 m n-6 n) x^{3} y^{6}, \\
& D_{y} D_{x} f(x, y)=6(4 m+4 n+4) x y^{6}+12(12 m n+8 m+8 n-4) x^{2} y^{6}+18(6 m n-6 n) x^{3} y^{6}, \\
& S_{x} S_{y}(f(x, y))=\frac{1}{6}(4 m+4 n+4) x y^{6}+\frac{1}{12}(12 m n+8 m+8 n-4) x^{2} y^{6}+\frac{1}{18}(6 m n-6 n) x^{3} y^{6}, \\
& D_{x}^{\alpha} D_{y}^{\alpha}(f(x, y))=6^{\alpha}(4 m+4 n+4) x y^{6}+2^{\alpha} \cdot 6^{\alpha}(12 m n+8 m+8 n-4) x^{2} y^{6}+3^{\alpha} \cdot 6^{\alpha}(6 m n-6 n) x^{3} y^{6}, \\
& S_{x}^{\alpha} S_{y}^{\alpha}(f(x, y))=\frac{1}{6^{\alpha}}(4 m+4 n+4) x y^{6}+\frac{1}{2^{\alpha} \cdot 6^{\alpha}}(12 m n+8 m+8 n-4) x^{2} y^{6}+\frac{1}{3^{\alpha} \cdot 6^{\alpha}}(6 m n-6 n) x^{3} y^{6}, \\
& S_{y} D_{x}(f(x, y))=\frac{1}{6}(4 m+4 n+4) x y^{6}+\frac{1}{3}(12 m n+8 m+8 n-4) x^{2} y^{6}+\frac{1}{2}(6 m n-6 n) x^{3} y^{6}, \\
& S_{x} D_{y}(f(x, y))=6(4 m+4 n+4) x y^{6}+3(12 m n+8 m+8 n-4) x^{2} y^{6}+2(6 m n-6 n) x^{3} y^{6}, \\
& S_{x} J f(x, y)=\frac{1}{7}(4 m+4 n+4) x^{7}+\frac{1}{8}(12 m n+8 m+8 n-4) x^{8}+\frac{1}{9}(6 m n-6 n) x^{3} y^{6}, \\
& S_{x} J D_{x} D_{y} f(x, y)=\frac{6}{7}(4 m+4 n+4) x^{7}+\frac{3}{2}(12 m n+8 m+8 n-4) x^{8}+2(6 m n-6 n) x^{3} y^{6}, \\
& S_{x}^{3} Q_{-2} J D_{x}^{3} D_{y}^{3} f(x, y)=\frac{216}{125}(4 m+4 n+4) x^{5}+8(12 m n+8 m+8 n-4) x^{6}+\frac{5832}{343}(6 m n-6 n) x^{7} .
\end{aligned}
$$

1. First Zagreb Index

$M_{1}(G)=\left.\left(D_{x}+D_{y}\right) f(x, y)\right|_{x=y=1}=150 m n+92 m+38 n-4$. 
2. Second Zagreb Index

$M_{2}(G)=\left.D_{y} D_{x}(f(x, y))\right|_{x=y=1}=252 m n+120 m+12 n-24$.

3. Modified Second Zagreb Index

${ }^{m} M_{2}(G)=\left.S_{x} S_{y}(f(x, y))\right|_{x=y=1}=\frac{4}{3} m n+\frac{4}{3} m+n+\frac{1}{3}$.

4. Generalized Randic' Index

$R_{\alpha}(G)=\left.D_{x}^{\alpha} D_{y}^{\alpha}(f(x, y))\right|_{x=y=1}=6^{\alpha}(4 m+4 n+4)+2^{\alpha} \cdot 6^{\alpha}(12 m n+8 m+8 n-4)+3^{\alpha} \cdot 6^{\alpha}(6 m n-6 n)$.

5. Inverse Randic' Index

$R R_{\alpha}(G)=\left.S_{x}^{\alpha} S_{y}^{\alpha}(f(x, y))\right|_{x=y=1}=\frac{1}{6^{\alpha}}(4 m+4 n+4)+\frac{1}{2^{\alpha} \cdot 6^{\alpha}}(12 m n+8 m+8 n-4)+\frac{1}{3^{\alpha} \cdot 6^{\alpha}}(6 m n-6 n)$.

6. Symmetric Division Index

$\operatorname{SSD}(G)=\left.\left(S_{y} D_{x}+S_{x} D_{y}\right)(f(x, y))\right|_{x=y=1}=55 m n+\frac{154}{3} m+\frac{109}{3} n+\frac{34}{3}$.

7. Harmonic Index

$H(G)=\left.2 S_{x} J(f(x, y))\right|_{x=1}=\frac{13}{3} m n+\frac{22}{7} m+\frac{38}{21} n-\frac{1}{7}$.

8. Inverse Sum Index

$I(G)=S_{x} J D_{x} D_{y}(f(x, y))_{x=1}=30 m n+\frac{108}{7} m+\frac{24}{7} n-\frac{18}{7}$.

9. Augmented Zagreb Index

$A(G)=\left.S_{x}{ }^{3} Q_{-2} J D_{x}{ }^{3} D_{y}{ }^{3}(f(x, y))\right|_{x=1}=\frac{67920}{343} m n+\frac{8864}{125} m-\frac{1333648}{42875} n-\frac{3136}{125}$.

\section{Conclusions}

In the present article, we computed closed form of M-polynomial for Bismuth TriIodide and then we derived many degree-based topological indices as well. Topological indices thus calculated for these Bismuth Tri-Iodide can help us to understand the physical features, chemical reactivity, and biological activities. In this point of view, a topological index can be regarded as a score function which maps each molecular structure to a real number and is used as descriptors of the molecule under testing. These results can also play a vital part in the determination of the significance of Bismuth Tri-Iodide.

For example, it has been experimentally verified that the first Zagreb index is directly related with total $\pi$-electron energy. Also Randic index is useful for determining physio-chemical properties of alkanes as noticed by chemist Melan Randic in 1975. He noticed the correlation between the Randic index $\mathrm{R}$ and several physico-chemical properties of alkanes like, enthalpies of formation, boiling points, chromatographic retention times, vapor pressure and surface areas. 


\section{Competing Interests}

The authors do not have any competing interests.

\section{Funding}

Funding is not available for this research.

\section{Data Availability}

Not Applicable

\section{References}

1. Imran, M., Ali, M. A., Ahmad, S., Siddiqui, M. K. \& Baig, A. Q. (2018). Topological Characterization of the Symmetrical Structure of Bismuth Tri-Iodide. Symmetry 2018, 10, 201; doi:10.3390/sym10060201

2. Iodide. B. McGraw-Hill Dictionary of Scientific and Technical Terms; McGraw-Hill: New York, NY, USA, 2003.

3. Mackay, R.A.; Henderson,W. Introduction to Modern Inorganic Chemistry; CRC Press: Boca Raton, FL, USA, 2002; pp. 122-126, ISBN 0-7487-6420-8.

4. Smart, L.; Elaine, A.M. Solid State Chemistry: An Introduction; CRC Press: Boca Raton, FL, USA, 2005; p. 40, ISBN 0-7487-7516-1.

5. Watanabe, K.; Karasawa, T.; Komatsu, T.; Kaifu, Y. Optical Properties of Extrinsic Two-Dimensional Excitons in BiI3 Single Crystals. J. Phys. Soc. Jpn. 1986, 55.

6. Wyckoff, R.W.G. Crystal Structures, 2nd ed.; John Wiley \& Sons, Inc.: New York, NY, USA; London, UK; Sydney, Australia, 1964.

7. Yorikawa, H.; Muramatsu, S. Theoretical Study of Crystal and Electronic Structures of BiI3. J. Phys. Condens. Matter 2008, 20, 325-335.

8. Nason, D.; Keller, L. The Growth and Crystallography of Bismuth Tri-Iodide Crystals Grown by Vapor Transport. J. Cryst. Growth 1995, 221.

9. G. Rucker, C. Rucker, On topological indices, boiling points, and cycloalkanes. J. Chem. Inf. Comput. Sci.1999, 39, 788-802.

10. S. Klavžar, I. Gutman, A Comparison of the Schultz molecular topological index with the Wiener index. J. Chem. Inf. Comput. Sci. 1996, 36, 1001-1003.

11. F. M. Brückler, T. Došli'c, A. Graovac, I. Gutman, On a class of distance-based molecular structure descriptors. Chem. Phys. Lett. 2011, 503, 336-338.

12. H. Deng, J. Yang, F. Xia, F. A general modeling of some vertex-degree based topological indices in benzenoid systems and phenylenes. Comp. Math. Appl.2011, 61, 3017-3023.

13. H. Zhang, F. Zhang, TheClar covering polynomial of hexagonal systems. Discret. Appl. Math. 1996, 69, 147-167. 
14. I. Gutman, Some properties of the Wiener polynomials. Graph Theory Notes New York1993, 125, 13-18.

15. E. Deutsch, S. Klavzar, M-Polynomial and degree-based topological indices. Iran. J. Math. Chem. 2015, 6, 93-102.

16. M. Munir, W. Nazeer,S. Rafique, S.M. Kang, M-polynomial and related topological indices of Nanostar dendrimers. Symmetry2016, 8, 97.

17. M. Munir, W. Nazeer,S. Rafique, A. R. Nizami, S.M. Kang, M-polynomial and degree-based topological indices of titania nanotubes. Symmetry 2016, 8, 117.

18. Kwun, Y. ,Munir, M. Nazeer, W; Rafique, S.; Kang, S. M. M-polynomial and degree-based topological indices of V-phenalinic nanotubes and nanotori, Scientific Reports 7, 8756, doi:10.1038/s41598-017-08309-y2017

19. M. Munir, W. Nazeer,S. Rafique, A. R. Nizami, S.M. Kang, Some Computational Aspects of Triangular Boron Nanotubes. Symmetry 2016, 9, 6; doi: 10.3390/sym9010006.

20. M. Munir, W. Nazeer,S. Rafique, S.M. Kang, M-Polynomial and Degree-Based Topological Indices of Polyhex Nanotubes. Symmetry 2016, 8, 149.

21. X. Yang, Y. Ding, and J. Ni, Phys. Rev. B 77, 041402(R) - Published 2 January 2008.

22. H. Wiener, Structural determination of paraffin boiling points. J. Am. Chem. Soc.1947, 69, 17-20.

23. A. A. Dobrynin, R. Entringer and I. Gutman,.Wiener index of trees: theory and applications. Acta Appl. Math. 2001, 66, 211-249.

24. I. Gutman, O. E. Polansky, Mathematical Concepts in Organic Chemistry; SpringerVerlag: New York, NY, USA, 1986.

25. M. Randic, On characterization of molecular branching,' J. Am. Chem. Soc. (1975), 97, 6609- 6615.

26. B. Bollobas, P. Erdos, Graphs of extremal weights. ArsCombin. (1998) 50, 225-233.

27. D. Amic, D. Beslo, B. Lucic, S. Nikolic, N. Trinajstic', The Vertex-Connectivity Index Revisited. J. Chem. Inf. Comput. Sci. (1998) 38, 819-822.

28. Y. Hu, X. Li, Y. Shi, T. Xu, I. Gutman, On molecular graphs with smallest and greatest zeroth-Corder general randic index. MATCH Commun. Math. Comput. Chem. (2005) 54, 425-434.

29. G. Caporossi, I. Gutman, P. Hansen, L. Pavlovic, Graphs with maximum connectivity index Comput. Biol. Chem. (2003) 27, 85-90.

30. X. Li, I. Gutman, Mathematical Chemistry Monographs, No. 1, Kragujevac, 2006.

31. L. B. Kier and L. H. Hall, Molecular Connectivity in Chemistry and Drug Research. Academic Press, New York, 1976.

32. L. B. Kier and L. H. Hall, Molecular Connectivity in Structure-Activity Analysis.Wiley, New York, 1986. 
33. X. Li and I. Gutman, Mathematical Aspects of Randic-Type Molecular Structure Descriptors. Univ. Kragujevac, Kragujevac, 2006.

34. I. Gutman and B. Furtula, Recent Results in the Theory of Randić Index. Univ. Kragujevac, Kragujevac, 2008.

35. M. Randić, On History of the RandićIndex and Emerging Hostility toward Chemical Graph Theory MATCH Commun. Math. Comput. Chem. 59 (2008) 5-124.

36. M.Randić, The Connectivity Index 25 Years After, J.Mol.Graphics Modell. 2001, 20, 19-35.

37. X. Li, Y. Shi, A survey on the Randic index. MATCH Commun. Math. Comput. Chem. 2008, 59, 127-156.

38. X. Li, Y. Shi, and L. Wang, in: Recent Results in the Theory of Randić Index, I. Gutman and B. Furtula (Eds.), Univ. Kragujevac, Kragujevac, 2008, pp. 9-47.

39. S. Nikolić, G. Kovačević, A. Miličević, and N. Trinajstić, The Zagreb indices 30 years after. Croat. Chem. Acta 76 (2003) 113-124.

40. I. Gutman and K. C. Das, The first Zagreb indices 30 years after, MATCH Commun. Math. Comput. Chem. 50 (2004), 83-92.

41. K. C. Das and I. Gutman, Some Properties of the Second Zagreb Index, MATCH Commun. Math. Comput. Chem. 52 (2004), 103-112.

42. N. Trinajstic, S. Nikolic, A. Milicevic, I. Gutman, On Zagreb indices, Kem. Ind. 59. (2010) 577-589 (in Croatian).

43. D. Vuki '̌evi'c, A. Graovac, Valence connectivities versus Randi'c, Zagreb and modified Zagreb index: A linear algorithm to check discriminative properties of indices in acyclic molecular graphs, Croat. Chem. Acta 77 (2004) 501-508.

44. Y. Huang, B. Liu, L. Gan, Augmented Zagreb Index of Connected Graphs, MATCH Commun. Math. Comput. Chem. 67 (2012) 483-494. 